\title{
Macrophage sequestration of HIV-1 enhances homeostatic-related systems in promoting viral spread and replication
}

\author{
Lawrence Agius \\ Department of Pathology, Mater Dei Hospital, University of Malta Medical School, Msida, Malta \\ Email: lawrence.agius@um.edu.mt, lawrence.agius@gov.mt
}

Received 7 March 2013; revised 7 April 2013; accepted 18 April 2013

Copyright (C) 2013 Lawrence Agius. This is an open access article distributed under the Creative Commons Attribution License, which permits unrestricted use, distribution, and reproduction in any medium, provided the original work is properly cited.

\begin{abstract}
Decisive modulatory systems of compromise and systems of dynamic turnover in lymphoid cells and macrophages are activated by repeated bursts of viremia and as promotional schemes of representation of subsequent spread and replication of HIV-1. In such operative systems of micro-environmental conditioning and reconditioning, a significant mechanism towards the turnover of specific cell-types occurs within context of sequestration within macrophages and circulating monocytes. Dendritic cells in germinal follicles and within specific organs such as the Langerhans cells of the skin are allied to dysfunctionality of such cellular subtypes as exemplified by the resident microglia of the central nervous system. Decisive perturbation in cell-type number and in dysfunctional activation indicate an exquisite modulatory role for HIV-1 in promoting homeostatic-related mechanisms within organs and tissues towards utilization in terms of viral dynamics and cytokine operability. In such manner, HIV-1 replication is itself a system of promotion in spread of viruses across cell-type and host cell specificities that tend to characterize and recharacterize systems of cytokine network operability in particular.
\end{abstract}

Keywords: HIV-1; Macrophages; Cytokine Networks; Endothelial Cells

\section{INTRODUCTION}

HIV-1 infection revolves around a systematic targeting of immune-competent cells in a manner that invokes normal homeostatic mechanisms in the furtherance of further progressive HIV-1 infection. Brain microvascular endothelial cells may regulate a central role in innate immu- nity to HIV infection by the blood-brain barrier [1]. It is with regard particularly of activation of the immune pathways that a whole plethora of effects constitutes the deliverance of detrimental consequences to antigen recognition and reactivity. It is beyond the simple institution of various defensive mechanistic steps that the immune system as integral whole proves extremely susceptible to depletion and qualitative and aberrant activation of cells that generally clear various other viral species from the circulation and from target infected cells.

HIV infection promotes an increase in production of free radicals and decreased production of glutaminecysteine ligase resulting in depletion of free glutathione with loss of innate immunity [2]. The overwhelmingly chronic replication of HIV-1 virions proves productive of lesions that compromise the turnover and equilibrium between distinct compartmentalized components of the immune system of the body.

\section{INCREMENTAL DETERIORATION}

Incremental deterioration of competent $\mathrm{T}$ cell $\mathrm{CD}^{+} 4$ subsets includes a concomitant viremic phase that contributes to set point parameters in inducing a conditioning series of adverse promoters to progression of replicative virion activity. Usage of CCR5 and CXCR4 receptors appears neither casual nor simply explained by their degree of cell surface expression [3].

Included in such scenario is the targeting mechanism in place that materially compromises the distinctive persistence of the HIV-1 infectious process itself. It is in terms of the ongoing parameters of lymphocyte and macrophage turnover in particular that contribute to the neuropathogenesis of an infection that evolves as basic endothelial cell participation within the central nervous system. In analogous fashion, the early involvement of gut-associated lymphoid tissue promotes a persistence of 
viral replication that is central to promotion of aberrant activation of lymphocytes. HIV protease inhibitor-induced inflammatory response in macrophages is a major risk factor for cardiovascular disease [4].

It is further to the multi-phasic promotion of injury to multiple subsets of the immune system ranging from T-lymphocytes, B cells, macrophages and circulating monocytes, and also dendritic cells and natural killer cells that there evolves an incongruity to the profile competency in clearance of replicating virus.

Retroviral infections tend to establish an equilibrium between virus replication and proviral latency in the infected host [5]. It is also in terms of distinctive retention of several response pathways on the part of the immune system that paradoxically promote an exquisite susceptibility to such lesions as opportunistic disease ranging from infections to neoplasia.

\section{DISTRIBUTIONAL DYNAMICS}

Distributional dynamics proves a determining series of mechanisms in effectively inducing lymphocyte turnover and pathway mechanics in promoting persistence of the viral infection. Toll-like receptors 7 and 8 in cellular endosomes bind single-stranded RNA of viral genomes to trigger intracellular signalling, inducing inflammatory cytokines and antiviral innate immunity [6].

Targeting of select subsets of lymphocytes is only one aspect in deregulating the immune pathways in a manner that globally promotes the intervention of further subsets in lesion promotion and persistence, particularly within the immune system itself. Dendritic cells and their subsets at the mucosal surfaces are amongst the first immune cells to encounter disseminating HIV-1 virus [7].

Significant participation of death pathways is illustrated by extensive apoptosis of lymphoid cells in a manner analogous to the parametric re-establishment of a series of factors that favor the persistence of spread of the virus. A major difference between immunocompetent individuals with Hodgkin's disease and individuals with HIV-associated classic Hodgkin's disease is the presence of necrotic foci and absence of epithelioid cells in the latter with a decrease in $\mathrm{CD} 4^{+}$T-cells [8].

It is significant to view spread of HIV-1 within contexts of shifting dynamics as well illustrated by involvement of dendritic cells in various organs ranging from lymphoid follicles to Langerhans cells to paracortical regions of lymph nodes and to gut-associated lymphoid tissue. HIV-1 subtype B replication in the CNS can occur in $\mathrm{CD}^{+} \mathrm{T}$ cells or macrophages/microglia in adults [9].

\section{NEUROPATHOGENESIS}

The emergence of neuropathogenic pathways is illustrative phenomenon as well-exemplified by the perivascular distribution of infected macrophages within the central nervous system. Cytokine networks globally participate in creating a multi-system promotion of viral persistence that proves conducive to further significant propagation of injury to immune cells themselves.

Cathepsins and cystatins are lysosomal proteins secreted by macrophages and microglia and may play essential roles in neuroregulatory systems [10].

Multi-focality is an essential aspect of HIV-1 replicative activity in terms of induced suppression in clearance of the virus. The distributional dimensions of infection correspond to the later susceptibility patterns of opportunistic infections that tend to characterize the advanced stages of AIDS.

It is further to such promotional cooperative systems of operative suppression of immunity that an essential permissive series of mechanisms proves effective in inducing active incorporation and integration of more provirus within the cellular genome. Even though HIV replication is significantly inhibited by interferon pretreatment, the virus is able to downregulate the transcription of known antiviral interferon-stimulated genes [11]. It is indeed significant to consider HIV-1 persistence of infection as essential virion replication in the first instance.

With reference to such dynamics, the spread and redistribution of lesions create a micro-environment that self-promotes adaptive depletion of immune cells. Quantitative and qualitative pathway lesions are well-recognized parameters in conjunction with repetitive bursts of viremia that re-establish effective viral persistence of replicative activity. Delivering adequate levels of drug to macrophages is important to reduce and eradicate HIV-1 infection [12].

\section{VIRION REPLICATION}

Virion replication is itself a determinant in conditioning the parameters of the viral infection within contextual reference of systems of participants such as cytokine operability. The significance of active dynamics of such virion replicative activity determines the outcome of systems of depletion in the first instance.

While all retroviruses replicate in dividing cells, lentiviruses uniquely replicate in non-dividing cells such as macrophages, linking cellular deoxynucleoside triphosphate levels and lentiviral adaptation intracellularly [13].

Modeling studies indicate a series of attempts at reconstitution of lymphoid pathways that prove detrimental to the homeostatic maintenance and mounting of immune responses to the virus. The whole micro-environment of infected cells as integral systems are conducive to an effective incorporation of virion integration within the cellular genome in a progressive and relentlessly aberrant manner. Endothelial cell dysfunction is one major mechanism involved in cardiovascular disease risk in the HIV patient [14]. One might consider the parameters of the 
viral integration as genomic participants in attempted reconstitution of systems of depletion.

\section{ABERRANT ACTIVATION}

Aberrant activation involves the cooperative involvement of lesions that are themselves active participants in retargeting of lesions. It is within specific conformational and steric hinderance systems of receptor-ligand cooperativity that cytokines are persistently reactivated by the HIV-1 infection.

Cooperative participation of cellular injury incorporates a distributional phenomenon in its own right and within systems of promotional facilitation of pathway lesions that are end-results in cell cycle dynamics. Monocytes, macrophages, and dendritic cells play an essential role in promoting transmission and spread and also as viral reservoirs in HIV-1 infection [15]. The distinctive specificities of injury include the apparent creation of syncytia of cells that characterize the incorporation of virus particles as distributional dynamics per se.

In this regard, participation of turnover of cells is a fundamental attribute in characterizing injury to an integral immune system that is essentially self-specific for individual organs within the body, as well illustrated by the central nervous system.

Transgression of organ individuality is essentially a reflection of HIV-1 infection within the specific confines of a micro-environment that proves essentially a conducive parameter in viral spread and replication. Chronic infected macrophages can account for the progression to AIDS through an initial acute infection, a long asymptomatic period and a final increase in viral load [16].

\section{VIRAL LOAD}

Inducement of the self-promotional increments in viral load is a significant correlative phenomenon in the establishment of injury as depletion of otherwise aberrantly activated lymphoid cells.

Profiles of aberrant reconstitution of the lymphoid system appear a particularly specific parameter in progression of injury as replicative virion phenomena. In such manner, the significant viremic phases in an HIV-1 infected individual are parameters of re-distribution that essentially correlate with distinctive stages in the establishment of relentless progression in persistent virion integration within the cellular genome.

Macrophages are reservoirs of active infection that recondition the viral dynamics in adapting to systems of cooperative participation in further events as cellular fusion and re-entry within lymphoid cells. The proportional dimensions of the reservoir populations of the virus are reflective of an ongoing endless series of reconditioning that incorporate distributional dynamics as well.
Within macrophages the HIV-1 regulatory protein "Nef" modulates surface receptors and signaling pathways and promote lymphoma, metabolic disease and neurologic disorders [17].

It is within the context of such promotional events as macrophage sequestration of virus that HIV-1 proves a persistent viral form of infection that is not cleared effectively even in the early stages of infection. Promotional emergence of viral budding and the viremic phases in the clinical course in HIV-1 infected individuals are systems that cooperatively prove relentless as part of aberrant homeostatic mechanisms in their own right. Monocytederived macrophages are more susceptible to infection and more permissive to HIV-1 replication than monocytes and may include the effects of different populations of miRNAs in these two cell groups [18]. The parametric reactivation of viral replication appears a real and constitutive component in a series of mechanistic processes that allow permissive conditions to dominant the infective phenomenon.

\section{REDISTRIBUTIONS OF VIRUS}

It is further to such promotional events that distribution of virions is a repeated series of redistributions of dynamic turnover of events as conditioned particularly by the viral sequestration events within the macrophages. The preferential utilization of protein disulfide isomerase may relate to HIV-1 entry and establishment of virus reservoirs in resting $\mathrm{CD} 4^{+}$cells while elevated thiredoxin levels in the chronic infection may facilitate entry in macrophages to maintain high viremia during the decline in T lymphocyte levels [19].

Significant overlap of antigenic profiles is another facet in the incremental viral spread of the injury inflicted within the micro-environment. It is within systems of cooperative suppressive effect that HIV-1 proves particularly adaptive in reconditioning pathways as promotional phenomena in spread of the viral particles.

Dynamics of macrophage migration and modulation of adhesion endothelial molecules indicate a realization that contributes to the development of ingress and egress movement within and between organs of specific functionality and anatomy. HIV-1 indicates a whole spectrum of selective participations that include the distributional models of spread as overall indicated by multiple bursts of viremia.

\section{RECONSTITUTION}

Proportional reconstitution of events of infection and replication indicate that these two parameters of progression are intimately correlated with systems of possible sequestration within specific organs. Such sequestration may possibly enhance realization of selectivity to host 
parenchymal cells as seen with the gut-associated lymphoid tissue and in regional lymphnodes.

In contrast to activated $\mathrm{CD} 4^{+} \mathrm{T}$ cells and differentiated macrophages, resting $\mathrm{CD}^{+}{ }^{+} \mathrm{T}$ cells and monocytes are non-permissive for HIV-1 replication, possibly implicating microRNAs that inhibit expression of cellular proteins and also viral co-factors [20]. Significant cellular interactivity contributes as networks of cytokine operability in inducing a micro-environment reconditioning in further promoting modulatory events in viral mutability. The various conformational adaptabilities allow for the promotion of sequestration on the one hand and an increment in distributional dynamics that are contributory phenomena in turnover of viral quasispecies within organs and tissues. Progressive immunodeficiency creates conditions in which viral evolution results in viral variants that target new cell types to create further classes of opportunistic infection [21].

Interchange between different cell types allow for sequestration that preconditions and further promotes distribution and spread of HIV-1 as evidenced in neural tissues.

The realization of evidential systems of co-operability may prove basic or generic mechanisms of modulatory adaptability as shown by the active sequestration of virus within macrophages and monocytes. HIV-1 endocytosis by macropinocytosis-like mechanisms leads to productive infection in macrophages; viral degradation is delayed following endosomal internalization allowing the virus to complete fusion [22]. The specificity of such dynamics may integrate within systems of overall promotion as shown by pathways of cellular dynamics within the circulatory system and also within the cellular microenvironment.

\section{CYTOKINES}

Cytokine activity and self-amplifying systems of dimensional or global participation operate within specific targeting systems of inducible modulation and as schemes of further promotion in viral replication and spread.

Inclusion of realized involvement of whole pathway profiles indicate the viral utilization of participating dynamics in further adjustment and cooperative adaptability in system modulation.

Host cells are included in the realization of events in such modulation in terms of overall involvement as evidenced by the cytokine networks that interactively promote or suppress such pathways as inflammatory reactivity. HIV-1 replication in macrophages is regulated by cytokines, and infection is restricted in macrophages activated by type 1 interferons and polarizing cytokines [23].

Reappraisal of multiple component pathways is directed as participants within the turnover dynamics of
HIV-1 that induce further modulation of cellular recaptivity, signalling and transduction and as cell trafficking pathways in further cellular response.

The progressive involvement of lymphoid cells in particular appears linked to a macrophage/monocyte adaptability in further enhancing the profiled viral progression in virion propagation.

\section{CONCLUDING REMARKS}

Overall dimensions of operability of lymphoid cells interact as microenvironmental systems of sequestration within macrophages and monocytes, on the one hand, and the responsive pathways elicited by parenchymal cells in various organs, on the other.

In such manner, distribution and patterned profiles of significant antigenic overlaps allow for the systematic organization of virion participation as evidenced by viral mutability.

In such terms, the incremental depletion of lymphocyte subsets is an index of promotional significance in enhancing distribution and replication of HIV-1 within otherwise homeostatically conditioned compartments within organs and tissues. It is in terms of ongoing turnover of cell-types that the sequestration of macrophages proves a system series of pathways in modulating HIV-1 adaptability and pathogenicity.

\section{REFERENCES}

[1] Li, J., Wang, Y., Wang, X., Ye, L., Zhou, Y., Persidsky, Y. and Ho, W. (2013) Immune activation of human brain microvascular endothelial cells inhibits HIV replication in macrophages. Blood, 121, 2934-2942.

[2] Morris, D., Guerra, C., Khurasany, M., Guilford, F., Saviola, B., Huang, Y., et al. (2013) Glutathione supplementation improves macrophage functions in HIV. Journal of Interferon \& Cytokine Research, 33, 270-279. doi:10.1089/jir.2012.0103

[3] Vicenzi, E., Lio, P. and Poli, G. (2013) The puzzling role of CXCR4 in human immunodeficiency virus infection. Theranostics, 3, 18-25. doi:10.7150/thno.5392

[4] Zha, W., Wang, G., Xu, W., Liu, X., Wang, Y., Zha, B.S., et al. (2013) Inhibition of P-glycoprotein by HIV protease inhibitors increases intracellular accumulation of berberine in murine and human macrophages. PLoS One, 8, Article ID: e54349. doi:10.1371/journal.pone.0054349

[5] Vicenzi, E. and Poli, G. (2013) Novel factors interfering with human immunodeficiency virus-type 1 replication in vivo and in vitro. Tissue Antigens, 81, 61-71. doi:10.1111/tan.12047

[6] Buitendijk, M., Eszterhas, S.K. and Howell, A.L. (2013) Gardiquimod: A toll-like receptor-7 agonist that inhibits HIV type 1 infection of human macrophages and activated T cells. AIDS Research and Human Retroviruses, 29, 907-918. doi:10.1089/aid.2012.0313

[7] Blanchet, F.P., Stalder, R., Czubala, M., Lehmann, M., 
Rio, L., Mangeat, B., et al. (2013) TLR-4 engagement of dendritic cells confers a BST-2/tetherin-mediated restriction of HIV-1 infection to CD4+ T cells across the virological synapse. Retrovirology, 10, 24.

[8] Hartmann, S., Jakobus, C., Rengsti, B., Doring, C., Newrzela, S., Brodt, H.R., et al. (2013) Spindle-shaped CD163+ resetting macrophages replace CD4+ T-cells in HIV-related classical Hodgkin lymphoma. Modern Pathology. doi:10.1038/modpathol.2012.217

[9] Sturdevant, C.B., Dow, A., Jabara, C.B., Joseph, S.B., Schnell, G., Takamune, N., et al. (2012) Central nervous system compartmentalization of HIV-1 subtype C variants early and late in infection in young children. PLOS Pathogens, 8, Article ID: e1003094. doi:10.1371/journal.ppat.1003094

[10] Cantres-Rosario, Y., Plaud-Valentin, M., Gerena, Y., Skolasky, R.L., Wojna, V. and Melendez, L.M. (2013) Cathepsin B and cystatin B in HIV-seropositive women are associated with infection and HIV-1-associated neurocognitive disorders. AIDS, 27, 347-356. doi:10.1097/QAD.0b013e32835b3e47

[11] Wie, S.H., Du, P., Luong, T.Q., Rought, S.E., BeliakovaBethell, N., Lozach, J., Corbell, J., et al. (2013) HIV downregulates interferon-stimulated genes in primary macrophages. Journal of Interferon \& Cytokine Research, 33, 90-95. doi:10.1089/jir.2012.0052

[12] Gavegnano, C., Detorio, M.A., Bassit, L., Hurwitz, S.J., North, T.W. and Schinazi, R.F. (2013) Cellular pharmacology and potency of HIV-1 nucleoside analogs in primary human macrophages. Antimicrob Agents Chemother, 57, 1262-1269. doi:10.1128/AAC.02012-12

[13] Amie, S.M., Noble, E. and Kim, B. (2013) Intracellular nucleotide levels and the control of retroviral infections. Virology, 436, 247-254.

[14] Lopez, M., San Roman, J., Estrada, V., Vispo, E., Blanco, F. and Soriano, V. (2012) Endothelial dysfunction in HIV infection-The role of circulating endothelial cells, microparticles, endothelial progenitor cells and macrophages. AIDS Reviews, 14, 223-230.

[15] Aggarwal, A., McAllery, S. and Turville, S.G. (2013)
Revising the role of myeloid cells in HIV pathogenesis. Current HIV/AIDS Reports, 10, 3-11. doi:10.1007/s11904-012-0149-1

[16] Hernandez-Vargas, E.A. and Middleton, R.H. (2013) Modeling the three stages in HIV infection. Journal of Theoretical Biology, 7, 33-40. doi:10.1016/j.jtbi.2012.11.028

[17] Lamers, S.L., Fogel, G.B., Singer, E.J., Salemi, M., Nolan, D.J., Huysentruyt, L.C., et al. (2012) HIV-1 nef in macrophage-mediated disease pathogenesis. International Reviews of Immunology, 31, 432-450. doi:10.3109/08830185.2012.737073

[18] Sisk, J.M., Clements, J.E. and Witwer, K.W. (2012) miRNA profiles of monocyte-lineage cells are consistent with complicated roles in HIV-1 restriction. Viruses, 4, 1844-1864. doi:10.3390/v4101844

[19] Stantchev, T.S., Paciga, M., Lankford, C.R., Schwartzkopff, F., Broder, C.C. and Clouse, K.A. (2012) Cell-type specific requirements for thiol/disulfide exchange during HIV-1 entry and infection. Retrovirology, 9, 97. doi:10.1186/1742-4690-9-97

[20] Chiang, K. and Rice, A.P. (2012) MicroRNA-mediated restriction of HIV-1 in resting CD4+ T cells and monocytes. Viruses, 4, 1390-1409. doi:10.3390/v4091390

[21] Swanstrom, R. and Coffin, J. (2012) HIV-1 pathogenesis: The virus. Cold Spring Harbor Perspectives in Medicine, 2, Article ID: a007443.

[22] Gobell, L.A., Lodge, R. and Tremblay, M.J. (2013) Macropinocytosis-like HIV-1 internalization in macrophages is CCR5 dependent and leads to efficient but delayed degradation in endosomal compartments. Journal of $\mathrm{Vi}$ rology, 87, 735-745. doi:10.1128/JVI.01802-12

[23] Jimenez, V.C., Boolman, T., de Taeye, S.W., van Dort, K.A., Rits, M.A., Hamann, J., et al. (2012) Differential expression of HIV-1 interfering factors in monocyte-derived macrophages timulated with polarizing cytokines or interferons. Scientific Reports, 2, 763. doi:10.1038/srep00763 